\title{
DETERMINATION OF LOW LEVEL RF CONTROL REQUIREMENTS FOR SUPERCONDUCTING CAVITIES FROM MICROPHONICS MEASUREMENTS *
}

\author{
J. R. Delayen, L. H. Harwood ${ }^{\#}$ \\ Thomas Jefferson National Accelerator Facility, Newport News, VA 23606
}

\begin{abstract}
In lightly beam loaded superconducting accelerators the rf power requirements are dictated by the maximum amount of microphonics that the system must be able to control and by the energy content of the cavity. In previous machines, such as heavy-ion boosters, the energy content of the cavities was relatively small and one could afford to specify a large control window and still use low power $(\sim 100 \mathrm{~W}) \mathrm{rf}$ sources. In newer accelerators that are being contemplated - such as the CEBAF $12 \mathrm{GeV}$ upgrade or RIA - the energy content of the cavity is quite large requiring rf sources in excess of $10 \mathrm{~kW}$. Conservatism in the control window specification would be quite costly; on the other hand loss of lock could have adverse effect on machine availability or beam property or even, in some cases, result in activation. We present a model to estimate the requirements to be placed on the rf control system based on the properties of the cavities, the frequency tuning system, and the characteristics of the accelerator.
\end{abstract}

\section{OVERVIEW}

Linacs based on superconducting RF (SRF) technology present novel optimization challenges to system designers. This is particularly true when the cavities are lightly beam loaded. In these cases the required RF power is not determined by the beam but rather by how much is required for phase and amplitude control in cavities whose resonant frequency deviates from the rf system's frequency. The amount of allowed detuning (deviation from the rf frequency) is strongly coupled to the expectations on the system's stability. A simple solution to the problem is to increase the available rf power; this has the negative effect of increasing the system cost. We describe below a methodology for projecting system performance utilizing a straightforward parameterization of the system parameters. This methodology then permits evaluation of trade-offs between system cost and system performance.

\section{BASIC REQUIREMENTS}

The generator power $P_{g}$ required to operated a cavity in the presence of beam loading and detuning is given by

$$
P_{g}=\frac{P_{c}}{4 ?}\left\{(1+?+b)^{2}+\dot{\varepsilon}(1+?) \tan ?-b \tan ? \dot{घ}^{2}\right\}
$$

\footnotetext{
*This work was supported by the U.S. Department of Energy Contract NumberDE-AC05-84-ER40150

\# delayen@jlab.org and harwood@jlab.org
}

where $P_{c}=V_{c}^{2} / R_{s h}$ is the power dissipated in the cavity walls at voltage $V_{c}$; ? is the usual coupling coefficient; $b=R_{s h} I \cos ? / V_{c}$ is the ratio of power absorbed by the beam and the power dissipated in the cavity walls; ? is the phase of the beam with respect to the rf; $\tan ?=-2(1+?) ? ? / ?{ }_{0}$ is the ratio of the frequency detuning and half the loaded bandwidth. Given the operational parameters of the cavity (voltage, beam current and phase), there is an optimal coupling coefficient ? that minimizes the generator power $P_{g}$ required to accommodate the frequency detuning ?? . At this optimal coupling, the generator power increases roughly linearly with the maximum amount of detuning that needs to be accommodated.

If the actual detuning exceeds what was assumed, the available power will not be sufficient to maintain control of the phase and amplitude of the cavity's field. When this occurs, the system is said to "lose lock" and the amplitude and phase of the cavity field will deviate from their set values resulting in a change in the energy gain provided by the cavity to the beam. If the shortfall is brief and/or is small, then the problems caused by the acceleration error may be minor. If the shortfall persists, the problems could be sufficiently large that other systems cannot correct for them. This may also result in beam impingement in some part of the accelerator which, in some circumstances, may not be acceptable.

\section{MODEL FOR THE AMOUNT OF TIME CAVITIES ARE OUT OF LOCK}

\subsection{Basic parameters}

Everything else being constant, there is a one-to-one relationship between the available rf power from the generator and the amount of detuning that can be accommodated. We will refer to this as the "detuning budget", ?budget.

Most SRF cavities have a resonance control system, typically called a "tuner", to mechanically adjust the cavity so that its average resonant frequency, ?cavity, matches the rf frequency, ? ${ }_{0}$. The control algorithms for the tuners usually do not activate the tuner until the cavity's frequency deviates from the $\mathrm{rf}$ frequency for more than some value, ? tuner. There is no physical reason

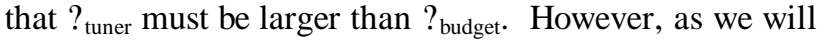


show later, the system performance is rather unsatisfactory if ? tuner $_{\text {is even comparable to ? } \text { budget }}$

A description is needed for the time distribution of

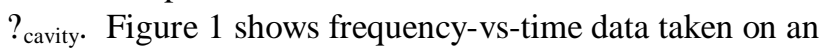
SNS cavity at JLab [1]. A simple characterization of the data would be that of a high frequency "noise" superimposed on a slow drift.
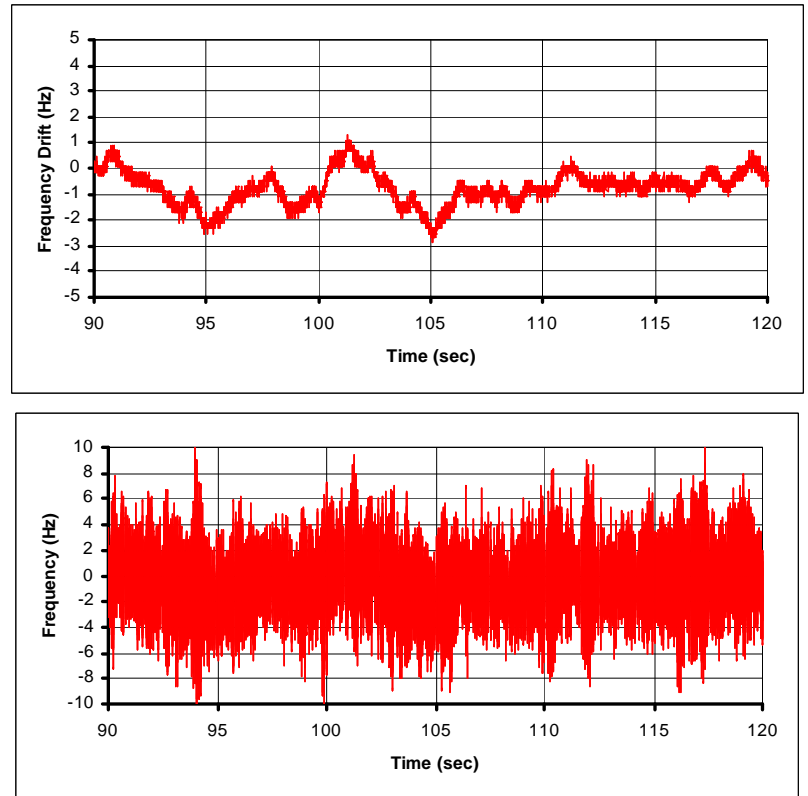

Figure 1:Frequency vs time measured for an SNS 805 $\mathrm{MHz}$ cavity at JLab during a 30 second period. The upper graph shows data using a $1 \mathrm{~Hz}$ low-pass filter; the data in the lower graph did not have the filter.

A natural approach would be to separate the distribution of frequency excursions into two classes, i.e. slow and fast. The slow events are those in which ? cavity changes slowly enough for the tuner control to respond; the fast ones are those where the tuner is unable to respond and need to be accommodated by the rf control system. In other words, we will use one description for the "noise" and another for the centroid of the "noise".

? "Noise": A probability density analysis of the data in Figure 1 yields a curve that is well characterized by a Gaussian. This data is consistent with the character of data for the 338 SRF cavities installed in CEBAF. In general, the width of the distribution, will depend on the specific cavity and cavity environment. The probability density associated with Fig. 1 is shown in Fig. 2.

? Slower events: the centroid of the Gaussian may not be static. Various options are available.

? One is to be very optimistic and assume that the centroid stays at center of the tuner range.

? Another is to assume that the centroid has equal probability to be anywhere between $?_{0^{-}} ?_{\text {tuner }}$ and $?_{0}+?_{\text {tuner }}$.

? A third is that the centroid undergoes a random walk from the origin. In this model the location of the centroid from its starting point since the last tuner operation would evolve as $t^{1 / 2}$, and the probability density for the centroid location would be quadratic inside the tuner window.

? And, finally, things could conspire to leave the centroid at the edge of the tuner window.

There are, of course, many other options, but the ones listed here likely span the range of credibility. The

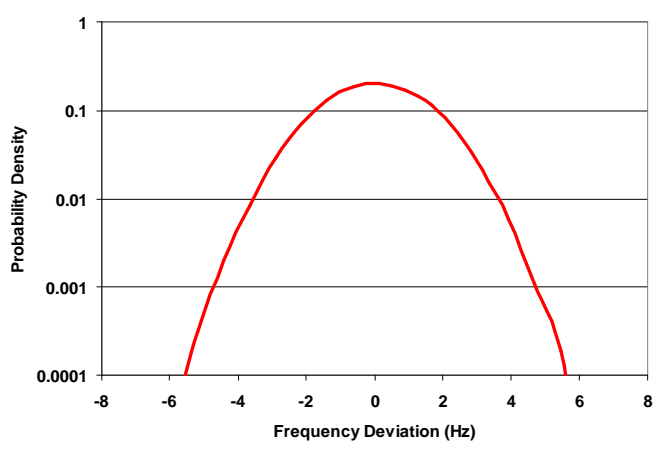

Figure 2: Probability density of the microphonics-induced frequency deviations. This was obtained from 400000 measurements of the instantaneous cavity frequency over an $800 \mathrm{sec}$ time span.

overall picture is illustrated in Figure 3.

Figure 3: Illustration of segmentation of frequency spectrum and "noise" spectrum (as represented by a Gaussian).

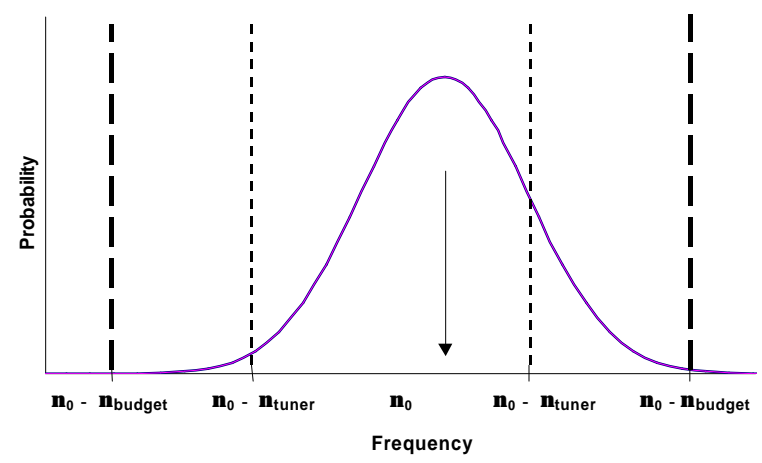

With choices for ?, ? cavity, ?tuner, and ? budget $_{\text {we can }}$ calculate the fraction of the time the system would be outside the ??? ${ }_{\text {budget }}$ to ???? ${ }_{\text {budget }}$ band. After calculating these probabilities for all/some values of ? cavity between 
????tuner and ????tuner. Next we select a model for the centroid drift and apply the appropriate weightings for that model to the probabilities we have calculated for each value of ? cavity. The result is the time averaged probability that the system will be "out of lock" within the assumptions of the model and for those values of the parameters.

\subsection{Results of the model}

Perhaps a more useful approach here is to generalize the problem by recasting it with dimensionless parameters rather than the previously described physical ones. First we note that it is not the absolute frequencies that are important, but rather their difference from $?_{0}$; we therefore shift the frequency axis by subtracting $?_{0}$ from all frequencies. Now the only quantities that enter the calculation are: ?, ?' cavity $\left(=\right.$ cavity $\left.?_{0}\right), ?_{\text {tuner }}$, and $?_{\text {budget }}$. We now shift to dimensionless parameters by dividing all of the quantities in the previous sentence by ? budget. The results are shown in Figure 4.
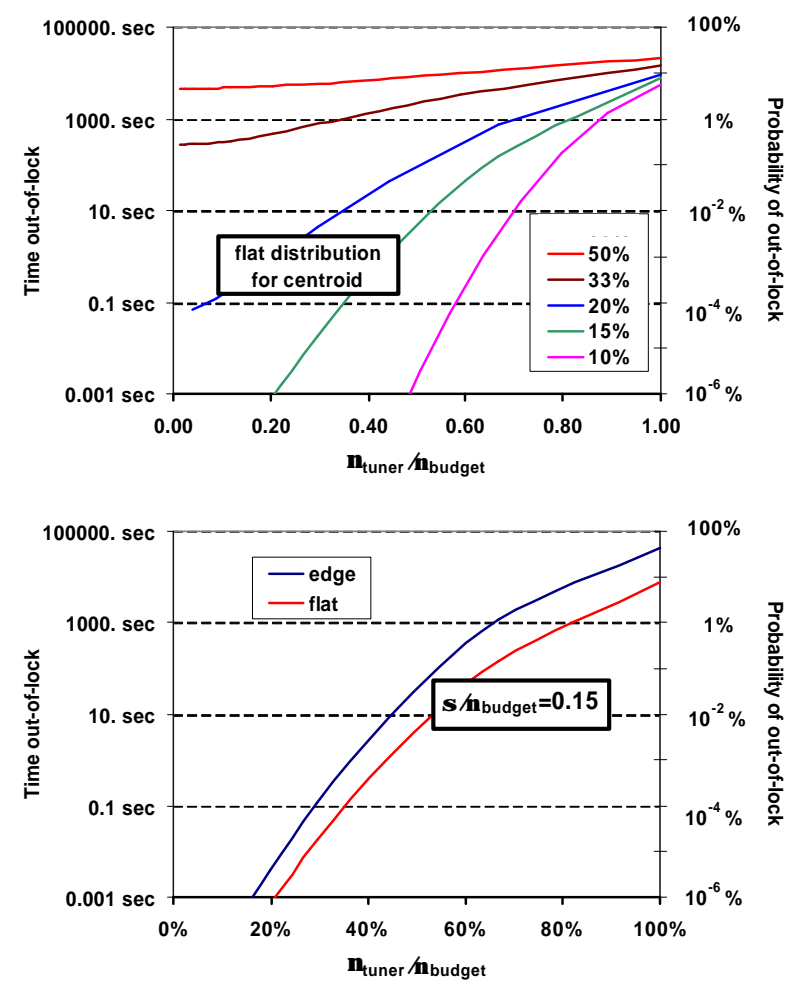

Figure 4: Probability of a cavity being out-of-lock and corresponding time per day of being out of lock. The upper graph shows the results for the flat distribution of and various values of ?/? budget. The lower graph shows the results for ?/? ${ }_{\text {budget }}$ with both the flat distribution and with the centroid sitting at budget $_{\text {. }}$
As can be seen, the probability of a cavity being out of lock is strongly dependent on both ?/? budget and $?_{\text {tuner }} / ?_{\text {budget }}$ ratios. One can also see that it is much less dependent on the choice for the distribution of the centroid.

\subsection{Limitations of the model}

The choice of a Gaussian to represent the "noise" distribution is an oversimplification of reality. In the data taken at Jefferson Lab, the "out of lock" time was best described by a Gaussian (whose characteristics could be determined from data taken over a fairly brief period of the order of $15 \mathrm{mn}$ ) with a few rare events superimposed on it. Thus, in practice if one chooses to use the Gaussian model, then it is imperative to acquire data over a long period, i.e. at least a day and preferably longer, when determining the ? to use. And alternative would be to use the ? from a short duration measurement and then assume that there will be some events that lead to additional "out of lock" time. It is also possible that there could be a strong source of mechanical noise that at a mechanical resonance of the cavity which could drastically distort the probability distribution away from a Gaussian.

\subsection{Extension beyond a single cavity}

The data shown in Figure 4 is for a single cavity and few systems have a single cavity. Predicting the net effects when there are several/many cavities becomes even more situationally dependent. For example, all the cavities could be acting completely independently; in this case one would simply multiply the probability for a single cavity by the number of cavities. On the other hand, they could all be affected by a single source of noise, e.g. the cryogenics system. Then the probability for the ensemble is close to that of a single cavity.

\section{MITIGATIONS}

As was mentioned earlier, the effect of going out of lock is that the cavity's gradient and phase wander. Should this occur, the quality of the beam will degrade. It is possible that the quality will degrade sufficiently and cause beam loss somewhere in the accelerator. Mitigating the degradation could be imperative.

It is possible for a feedback system that monitors the linac energy gain to compensate for some of the out-oflock events; this is most tractable if there is only a small number of cavities that are out-of-lock. If, on the other hand, several cavities are out-of-lock, then it is quite unlikely that it would be possible to compensate for it.

Another option would be to temporarily provide extra rf power or, at least, reduce the total rf power load. The former option might be possible if there is something, e.g. a modulating anode in a klystron that is throttling the power below the maximum. The latter could be achieved by temporarily reducing the beam current or cavity voltage, thereby making available a larger portion of the rf power for control. 


\section{SUMMARY}

We have presented a methodology for predicting the amount of time an rf system will be out-of-lock which is consistent with measurements on real cavities. In addition, we have presented potential mitigations for those times when the cavities are out-of-lock. We believe both are valuable when planning the rf system for anticipated accelerators where the cost of $\mathrm{rf}$ power is a significant constraint.

\section{REFERENCE}

[1] Jean Delayen, Ed Daly, Kirk Davis, Steve Smee, "Frequency Measurements on the Prototype SNS Medium-? Cryomodule Under Pulsed and CW Operation," JLab Tech Note 02-049. 